DESY 00-044

ISSN 0418-9833

MZ-TH/00-09

hep-ph/0003148

March 2000

\title{
Gluon Fragmentation to Gluonium
}

\author{
H. Spiesberger ${ }^{1}$ and P. M. Zerwas ${ }^{2}$ \\ ${ }^{1}$ Institut für Physik, Johannes-Gutenberg-Universität, \\ Staudinger Weg 7, D-55099 Mainz, Germany \\ 2 Deutsches Elektronen-Synchrotron DESY, D-22603 Hamburg, Germany
}

\begin{abstract}
The fragmentation of gluons to gluonium states is analyzed qualitatively in the nonperturbative region. The convolution of this mechanism with perturbative gluon radiation leaves us with a hard component in the fragmentation of gluon to gluonium.
\end{abstract}


Theoretical analyses of gluonic matter particles were initiated [1] soon after gluon fields were introduced as the basic force fields of the strong interactions. Mass spectra and quantum numbers of such states have been studied in several approaches to non-perturbative QCD [2]. Since gluon-rich environments of collision processes are the preferential source for the production of gluonia, several mechanisms of this kind have been analyzed at great detail, in particular heavy quarkonium decays [3] and Pomeron processes [4]. Strong candidates have been observed in various experimental analyses [5], though final conclusions could not be drawn yet.

Recently it has been suggested to search for gluonium states in $Z$ decays [6]. A significant fraction of these decays involves gluon jets so that the LEP/SLC $Z$ events provide a natural ensemble to search for such states. The energy of these gluon jets is large enough to justify a parton picture for the production of gluonia. This process can be described by the convolution of the hard cross section for producing a gluon with a fragmentation function accounting for the transition of gluons to gluonia. The resulting expressions are correct up to terms which decrease as inverse powers of the gluon-jet energy.

In this note we describe an attempt to predict the momentum spectrum of the gluonium particles within the fragmented gluon jets. The solution of this problem is of experimental relevance in optimizing search strategies for gluonia. In particular, if a major fraction of the gluon energy is transferred to the gluonium states in the fragmentation process, the experimental identification is facilitated vis-a-vis the soft fusion of gluons to gluonia in the plateau region for which the gluonium decay products submerge to the low-energy hadron sea.

Non-perturbative Fragmentation: The basic idea for the primordial non-perturbative fragmentation of gluons to generic gluonium statesf $G$ of mass $M_{G} \simeq 1.5 \mathrm{GeV}$ follows the path of heavy-quark fragmentation, the gross structure of which can be described by the Peterson et al. fragmentation function [7]. A simple form of the fragmentation function can be derived by adopting the quantum-mechanical rules of old-fashioned perturbation theory [8] to estimate transition probabilities in the parton model. The qualitative features of the amplitude for a high-energy gluon $g$ to fragment into a gluonium state $G$ with a fraction $z$ of the gluon momentum are determined by the energy transfer $\Delta E=E_{G}+E_{g}^{\prime}-E_{g}$ across

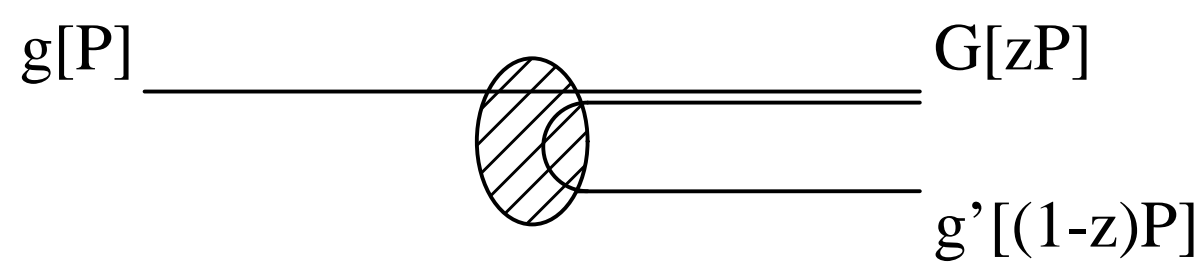

Figure 1: The non-perturbative $g \rightarrow G$ fragmentation in the parton picture.

\footnotetext{
${ }^{1}$ To analyze the gross features of the fragmentation function, it is sufficient to consider $g g$ gluonium states as example.
} 
the vertex in the fragmentation process (see Fig. 1) which conserves three-momentum:

$$
\text { Amplitude }\left[g \rightarrow G+g^{\prime}\right] \propto \Delta E^{-1}
$$

Expanding the energies for large gluon momenta $P$ about the gluonium mass $M_{G}$ and the transverse momentum which can be assumed of order of the strong interaction scale $\Lambda$,

$$
\begin{aligned}
\Delta E & =\sqrt{M_{G}^{2}+z^{2} P^{2}}+\sqrt{\Lambda^{2}+(1-z)^{2} P^{2}}-P \\
& \propto \frac{1}{z}+\frac{\epsilon_{G}}{1-z},
\end{aligned}
$$

and taking into account the standard factor $z^{-1}$ for the longitudinal phase space which generates the non-perturbative rapidity plateau, we suggest the following ansatz for the non-perturbative $g \rightarrow G$ fragmentation function:

$$
d_{g}^{G}(z)=\frac{N}{z\left(\frac{1}{z}+\frac{\epsilon_{G}}{1-z}\right)^{2}} .
$$

The shape parameter $\epsilon_{G}$ is defined as

$$
\epsilon_{G}=\Lambda^{2} / M_{G}^{2}
$$

according to the expansion Eq. (2).

The coefficient $N$ denotes the normalization which after integration over the momentum spectrum defines the (unknown) rate at which gluons fragment non-perturbatively into gluonium states. Choosing for illustration $M_{G}=1.5 \mathrm{GeV}$ and $\Lambda=0.5 \mathrm{GeV}$, the small coefficient $\epsilon_{G} \simeq O(1 / 10)$ makes $d_{g}^{G}(z)$ peak strongly near $z=1$ (dashed curve in Fig. 2). The maximum of the momentum distribution is predicted at $z_{\max } \simeq 1-\sqrt{2 \epsilon_{G}}$ for small $\epsilon_{G}$. This form is a straightforward consequence of quantum mechanics which enhances transitions with minimum energy transfer. For large momenta, i.e. large $z$, the impact of the heavy gluonium mass on the energy transfer is less effective than for small momenta.

This fragmentation picture applies only for large energies of the fragmenting gluon for which $\Delta E \propto P^{-1}$ approaches zero. A lower bound of the required gluon energy may be estimated by demanding the energy transfer $\Delta E$ to be less than a fraction of the typical strong interaction scale $\Lambda$. It follows from the inequality $\Delta E \lesssim \Lambda / 2$ that the gluon energy must exceed

$$
E \gtrsim E_{0} \simeq M_{G}^{2} / \Lambda
$$

in the laboratory frame which amounts to about $5 \mathrm{GeV}$ for the parameters introduced above.

Perturbative Gluon Radiation: If the gluon energy exceeds the minimum value (5), the parent gluon is attenuated by secondary gluon bremsstrahlung at early times before the non-perturbative fragmentation becomes operative. These perturbative QCD effects can 


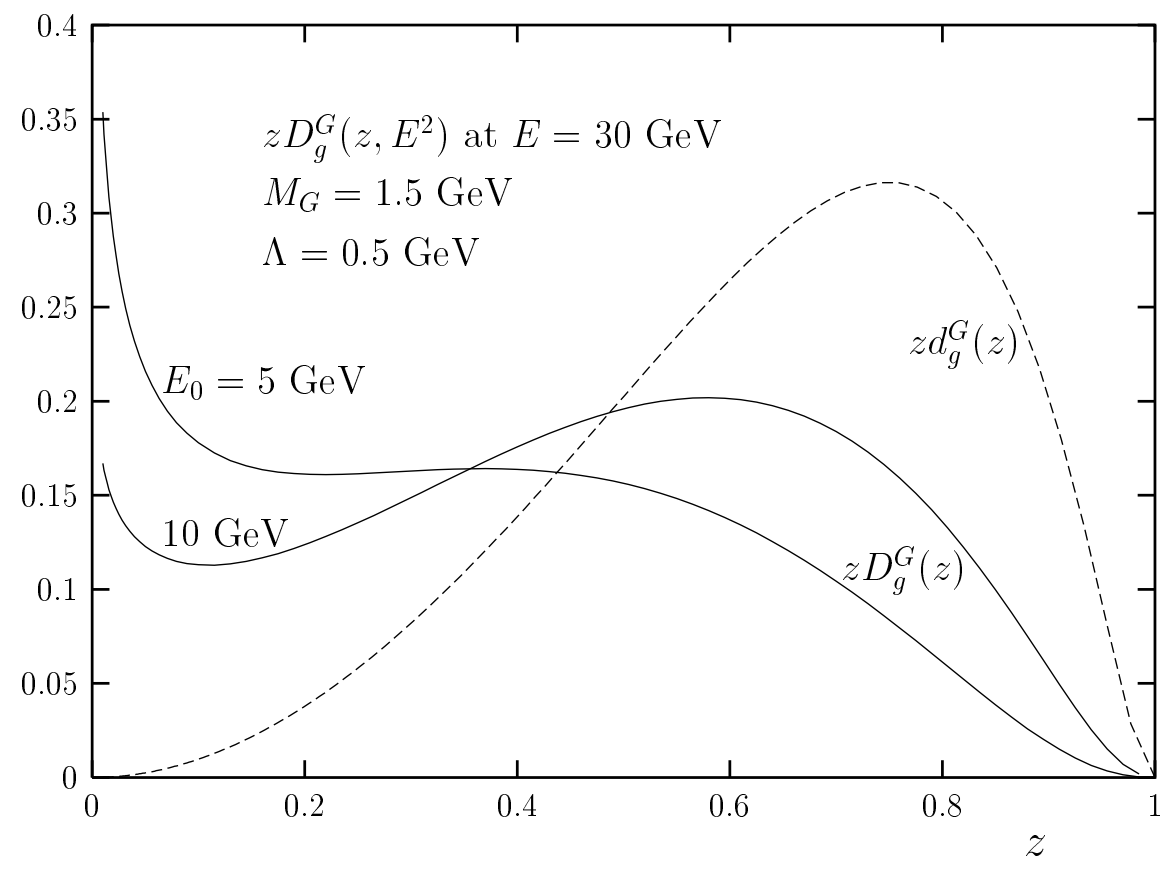

Figure 2: Gluon-to-gluonium fragmentation at the energy $E=30 \mathrm{GeV}$. Dotted line: non-perturbative fragmentation function $d_{g}^{G}(z)$ with $N=1$ (for the sake of clarity the fragmentation functions are multiplied with the momentum fraction $z$ ); full lines: fragmentation function $D_{g}^{G}\left(z, E^{2}\right)$ after the evolution from the scales $E_{0}=5$ and $10 \mathrm{GeV}$ to the energy $E=30 \mathrm{GeV}$.

be described in analogy to the Altarelli-Parisi evolution [9]. Neglecting the higher-order effect of quark feedback to gluons within the gluon jet, the attenuation is described in moment spacel by the coefficient

$$
g\left(m, E^{2}\right)=\left[\frac{\alpha_{s}\left(E^{2}\right)}{\alpha_{s}\left(E_{0}^{2}\right)}\right]^{2 \gamma_{m} / \beta_{0}}
$$

for the evolution from the energy $E_{0}$ to $E . \gamma_{m}$ is the anomalous dimension related to the gluon splittings $g \rightarrow g g, q \bar{q}$ :

$$
\gamma_{m}=\frac{3}{2}\left(-\frac{1}{3}-\frac{2 N_{F}}{9}+\frac{4}{m(m-1)}+\frac{4}{(m+1)(m+2)}-4 \sum_{j=2}^{n} \frac{1}{j}\right)
$$

with $\beta_{0}=11-2 N_{F} / 3$ where we take $N_{F}=4$ active light flavours.

Summary: The final gluon-to-gluonium fragmentation function $D_{g}^{G}$ at energy $E$ which incorporates the perturbative and non-perturbative effects is found by convoluting the

\footnotetext{
${ }^{2}$ The moments of the function $f(z)$ are defined by $f(m)=\int_{0}^{1} d z z^{m-2} f(z)$.
} 
perturbative splitting function with the non-perturbative fragmentation function; in moment space:

$$
D_{g}^{G}\left(m, E^{2}\right)=d_{g}^{G}(m)\left[\frac{\alpha_{s}\left(E^{2}\right)}{\alpha_{s}\left(E_{0}^{2}\right)}\right]^{2 \gamma_{m} / \beta_{0}}
$$

with $d_{g}^{G}$ describing the non-perturbative fragmentation process at the energy $E_{0} \simeq M_{G}^{2} / \Lambda$ as argued before.

Transformed back to momentum space, the fragmentation function $D_{g}^{G}\left(z, E^{2}\right)$ is illustrated for the gluon energy $E=30 \mathrm{GeV}$ by the full curves in Fig. 2. The fragmentation function is shown for two values of the initial energy $E_{0}$ for the definition of the nonperturbative function $d_{g}^{G}(z)$. The variation illustrates the inherent uncertainties due to the qualitative estimate of $E_{0}$.

However, despite of these quantitative uncertainties the qualitative picture emerges quite clearly: A hard component is expected to be present when gluonia are formed in the fragmentation of gluon jets. Gluonium particles will also be generated by gluon-gluon fusion mechanisms at low energies in the plateau region of the gluon jets. These additional mechanisms will increase the overall multiplicity of gluonia in the jet, yet they will not reduce the particle yield generated by the hard component in gluon fragmentation to gluonium at large $z$. The hard component may experimentally be quite helpful for the search of these novel particles since the reconstruction is easier in a phase space region of low hadronic population.

Acknowledgement: We thank P. Roy for an inspiring discussion on the search for gluonia in gluon fragmentation.

Note: After writing this letter, we received the paper of Ref. [10] in which elements of the production rate for gluonia in gluon fragmentation have been discussed.

\section{References}

[1] H. Fritzsch and M. Gell-Mann, Proceedings, XVI Int. Conference on High Energy Physics, FNAL 1972; H. Fritzsch and P. Minkowski, Nuovo Cim. 30A (1975) 393.

[2] M. Chanowitz and S. Sharpe, Nucl. Phys. B222 (1983) 211; T. Schafer and E. V. Shuryak, Phys. Rev. Lett. 75 (1995) 1707; G. S. Bali et al., (UKQCD Collaboration), Phys. Lett. B309 (1993) 378; D. Weingarten, Nucl. Phys. B (Proc. Suppl.) 34 (1994) 29.

[3] P. Roy and T. F. Walsh, Phys. Lett. B78 (1978) 62.

[4] F. E. Close and A. Kirk, Phys. Lett. B397 (1997) 333; ibid. B410 (1997) 353 (Erratum). 
[5] Particle Data Group, C. Caso et al., Eur. Phys. J. C3 (1998) 1; M. E. Pennington, Proceedings, Workshop on Photon Interactions and the Photon Structure, ed. G. Jarlskog and T. Sjostrand, Lund 1998, hep-ph/9811276].

[6] P. Roy and K. Sridhar, JHEP 9907 (1999) 13.

[7] C. Peterson, D. Schlatter, I. Schmitt and P. Zerwas, Phys. Rev. D27 (1983) 105.

[8] K. Nishijima, Fundamental Particles, Benjamin 1964.

[9] G. Altarelli and G. Parisi, Nucl. Phys. B126 (1977) 298.

[10] P. Minkowski and W. Ochs, e-preprint hep-ph/0003125. 